\title{
Location of the Galvanometer Branch for Maximum Sensitivity of the Wheatstone Bridge
}

\author{
By F. Ralph Kotter
}

\begin{abstract}
The battery and galvanometer connections to a Wheatstone bridge may be interchanged without altering the condition for balance. One of the combinations will give a higher sensitivity than the other, but the calculation of the better arrangement is often somewhat tedious. This paper presents a "rule-of-thumb" method of determining the better arrangement, which requires only a knowledge of the resistance of the bridge arms and the value of the external critical damping resistance of the galvanometer.
\end{abstract}

The battery and galvanometer connections to the Wheatstone bridge may be interchanged without affecting the condition of balance of the bridge. One of the arrangements will usually be more sensitive than the other.

The question of which connection would give the higher sensitivity was investigated by Maxwell. ${ }^{1}$ His conclusions, which have formed the basis for most subsequent treatments of the subject, were based on the assumption that the battery would be the limiting factor in the sensitivity, whereas now batteries that will supply more energy than can be dissipated as heat in the bridge arms are universally available. Wenner ${ }^{2}$ has recently discussed the problem of the connection of the galvanometer branch under conditions of modern laboratory practice, considering the limiting power in the bridge arms and assuming that the galvanometer is always used critically damped or with the same percentage of critical damping.

It has been found that Wenner's analysis may be extended to give a simple rule of thumb method for determining the better position for the galvanometer branch. This method requires only a knowledge of the resistances of the bridge arms and the value of resistance required to produce critical damping of the galvanometer. As will be subsequently proved, the rule ${ }^{3}$ to be followed is:

\footnotetext{
1 J. C. Maxwell, A treatise on electricity and magnetism 1, 400 (1873).

${ }_{2}^{2}$ F. Wenner, J. Research NBS $\mathbf{2 5}, 229$ (1940) RP1323.

3 The possibility that this rule is valid was suggested to the author by J. L. Thomas of this Bureau. A similar but less precise rule is given by $\mathrm{H}$. B Brooks in the section titled, Measurements and measuring apparatus, of Pender and Del Mar's Electrical engineer's handbook 4, section 5, p. 5, 3d ed. (John Wiley \& Sons, Inc., New York, N. Y., 1936).
}

Calculate the resistance of the bridge between each pair of opposite branch points with galvanometer and battery circuits open. Compute the ratio or the reciprocal ratio, whichever is less than unity, of each of these "bridge resistances" to the external critical damping resistance of the galvanometer. Connect the galvanometer to the pair of branch points for which the ratio is nearer unity.

The rule given above assumes (1) that the galvanometer is to be used with critical damping, (2) that the limitation on the maximum power is the same for each arm of the bridge, and (3) that the voltage applied to the bridge is sufficient to dissipate this amount of power in one arm and no greater amount in any arm of the bridge. Under these conditions the rule is perfectly general, applying to all values of bridge ratios, bridge arm resistances, and galvanometer external critical damping resistances.

Perhaps more frequently than not, it is desirable to use the galvanometer slightly underdamped. In that event the above rule applies if the required calculations are made using the value of external damping resistance that has been found desirable rather than the critical damping resistance.

Proof.-The validity of the rule stated above will be established by determining, for all possible values of the galvanometer external critical damping resistance, the relative sensitivities and relative values of the ratios of bridge resistances to damping resistance for the two possible locations of the galvanometer branch in a Wheatstone bridge. Subject to the conditions of balance, the ratios 
and the resistances of the bridge arms may have any desired values.

There is no loss in generality in assuming the bridge arms to have the values shown in figure 1 and requiring that $n>p \geqq 1$. (The case of $n=p$ is trivial, since the bridges $(a)$ and $(b)$ are then identical.)

The bridge resistances across the galvanometer for the two cases are

and

$$
W_{a}=\frac{n R(1+p)}{1+n},
$$

$$
W_{b}=\frac{p R(1+n)}{1+p} .
$$

It may be readily shown that $W_{b}>W_{a}$ for all values of $n$ and $p$ subject to the condition $n>p \geqq 1$ :

$$
\frac{W_{a}}{W_{b}}=\frac{n(1+p)^{2}}{p(1+n)^{2}} .
$$

Let $n=p+q,(q>0)$. Then

$$
\frac{W_{a}}{W_{b}}=\frac{p^{3}+2 p^{2}+p^{2} q+p+2 p q+q}{p^{3}+2 p^{2}+p^{2} q+p+2 p q+q\left(p^{2}+p q\right)} .
$$

As $q>0$ and $p \geqq 1$, the denominator is larger than the numerator and $W_{b}>W_{a}$.

The sensitivities and resistance ratios to be compared are continuous functions of the galvanometer critical damping resistance; however, the functions are each two joined straight lines, with different slopes for the two parts. This is illustrated in figure 2, where curve "bridge $a$ " shows

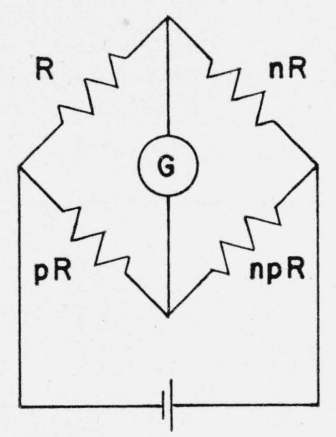

(a)

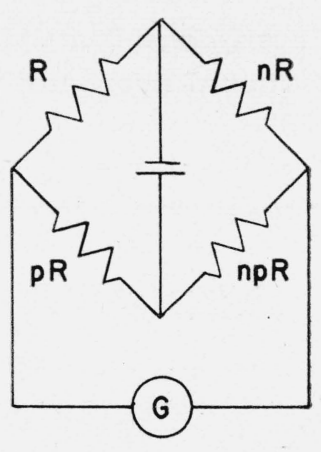

(b)
FiguRE 1. Alternate connections of battery and galvanometer in the Wheatstone bridge.

the variation of bridge sensitivity with galvanometer external critical damping resistance when the galvanometer is connected as shown in $(a)$ of figure 1. Curve "bridge $b$ " of figure 2 is the corresponding curve of sensitivity when the connection is that of $(b)$ in figure 1 . It is therefore necessary to carry out the proof in the following four cases:

Case $1, V \leqq W_{a}$ :

Let $V$ represent the critical external damping resistance for the galvanometer and consider first $V \leqq W_{a}$. The ratios to be compared are $V / W_{a}$ and $V / W_{b}$. fore

It has already been shown that $W_{b}>W_{a}$, there-

$$
\frac{V}{W_{a}}>\underset{W_{b}}{V}
$$

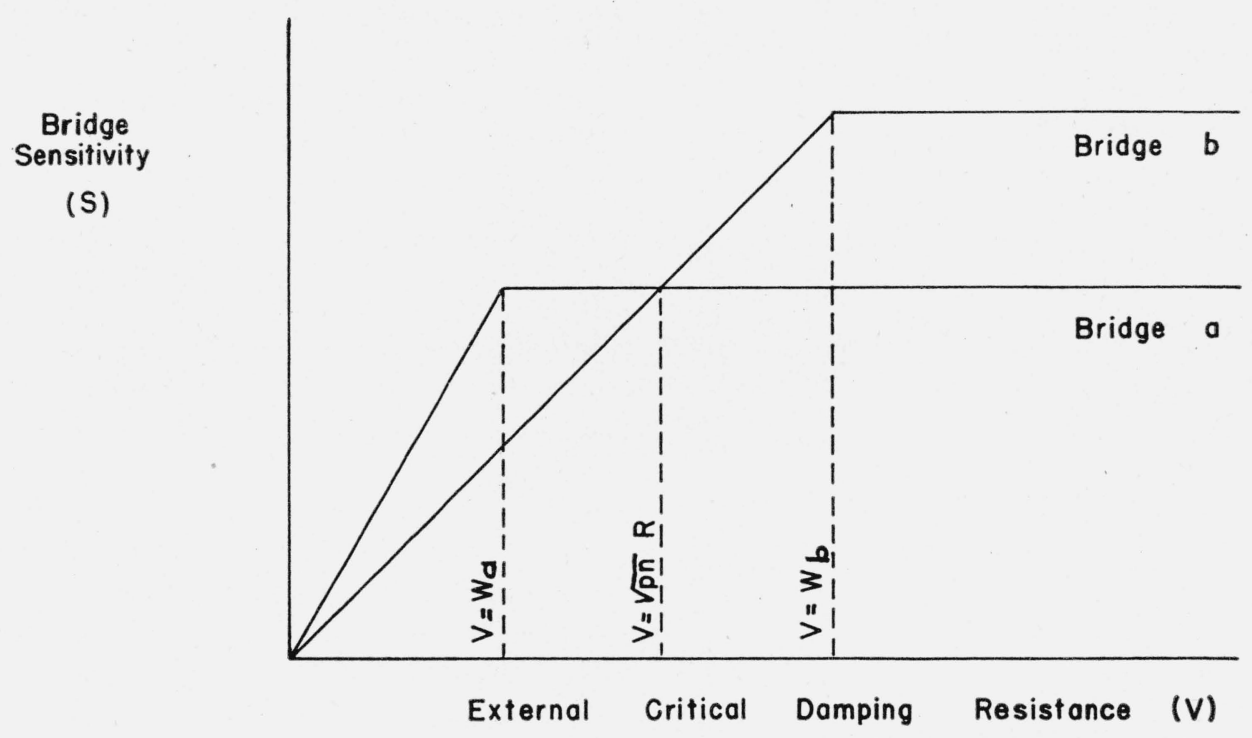

FIGURE 2. Variation of bridge sensitivity with galvanometer external critical damping resistance. 
Case $2, W_{a}<V \leqq \sqrt{p n} R$ :

Now consider $W_{a}<V \leqq \sqrt{p n} R$. The ratios to be compared are $W_{a} / V$ and $V / W_{b}$.

Dividing one ratio by the other and using eq. 1 and 2 gives

$$
\frac{W_{a} / V}{V / W_{b}}=\frac{p n R^{2}}{V^{2}}
$$

This ratio is equal to unity for $V=\sqrt{p n} R$ and greater than unity for all values of $V$ less than $\sqrt{p n} R$. Therefore,

$$
\frac{W_{a}}{V} \geqq \frac{V}{W_{b}} \text { for } V \leqq \sqrt{p n R} .
$$

Case $3, \sqrt{p n} R<V<W_{b}$ :

Now consider $\sqrt{p n} R<V<W_{b}$. The ratios to be compared are again $W_{a} / V$ and $V / W_{b}$, and eq. 4 shows that

$$
\frac{W_{a}}{V}<\frac{V}{W_{b}} \text { for } V>\sqrt{p n} R .
$$

Case $4, V \geqq W_{b}$ :

In the range $V \geqq W_{b}$ the ratios to be compared are $W_{a} / V$ and $W_{b} / V$.

It has already been shown that $W_{b}>W_{a}$, and consequently

$$
\frac{W_{a}}{V}<\frac{W_{b}}{V}
$$

It has now been shown that the ratio involving $W_{a}$ and $V$ is larger than the ratio involving $W_{b}$ and $V$ for all values of $V<\sqrt{p n} R$, that the ratios are equal for $V=\sqrt{p n} R$ and that the ratio involving $W_{b}$ and $V$ is larger than the one involving $W_{a}$ and $V$ for $V>\sqrt{p n} R$. It remains then to be shown that the sensitivity of bridge $a$ is greater than that of bridge $b$ for $V<\sqrt{p n} R$, that the sensitivities are equal for $V=\sqrt{p n} R$, and that the sensitivity of $b$ is greater than $a$ for $V>\sqrt{p n} R$.

Since the power is greatest in the $n R$ arm of the bridge of figure 1 a the resistance of this arm imposes the limitation on the voltage that may be applied to the bridge and Wenner's equation for the bridge sensitivity, ${ }^{4} S_{a}$, may be written

$$
S_{a}=2 D E_{n R} \frac{R}{R+n R} \times \frac{V}{W_{a}},
$$

where $D$ is the voltage sensitivity of the galvanometer in scale divisions per unit electromotive force when the resistance in series with the galvanome-

\footnotetext{
${ }_{4}^{4}$ F. Wenner, J. Research NBS 25, 238 (1940) RP1323.
}

ter is $V ; E_{n R}$ is the maximum permissible potential drop in $n R$; and the other symbols have the meanings previously given them.

As is shown by Wenner, when the ratio $V / W_{a}$ is less than or equal to unity its actual value is to be used in the equation for sensitivity; however, when the ratio $V / W_{a}$ is greater than unity, it is to be replaced by unity in the equation. This assumes, as stated above, that the galvanometer damping is always adjusted to the same value either by series or parallel resistance as required.

Expressed in terms of the permissible power, $P_{n R}$, in the $n R$ arm, the equation may be written

$$
S_{a}=2 D \sqrt{P_{n R}} \sqrt{n R} \times \frac{R}{R+n R} \times \frac{V}{W_{a}} .
$$

For the bridge in figure $1, \mathrm{~b}$, the limitation is imposed by the $p R$ arm, and in terms of the permissible power in it $\left(P_{p R}\right)$ the sensitivity may be written

$$
S_{b}=2 D \sqrt{P_{p R}} \sqrt{p R} \times \frac{R}{R+p R} \times \frac{V}{W_{b}} .
$$

As it is assumed that the power limitations are the same for all arms of the bridge, $P_{p R}=P_{n R}$, and eq 8 and 9 may be rewritten

$$
\begin{aligned}
& S_{a}=K \frac{\sqrt{n R}}{1+n} \times \frac{V}{W_{a}}, \\
& S_{b}=K \frac{\sqrt{p R}}{1+p} \times \frac{V}{W_{b}} .
\end{aligned}
$$

For values of $V$ equal to or less than $W_{a}$, the ratio $V / W_{a}$ is equal to or less than unity, $V / W_{b}$ is less than unity, and the actual values are to be used in eq 10 and 11.

Then for $V \leqq W_{a}$, using values of $W_{a}$ and $W_{b}$ from eq 1 and 2 ,

$$
\begin{aligned}
& S_{a}=\frac{K V}{\sqrt{n \bar{R}(1+p)}}, \\
& S_{b}=\frac{K V}{\sqrt{p \bar{R}(1+n)}}, \\
& \frac{S_{a}}{S_{b}}=\sqrt{\frac{p(1+n)^{2}}{n(1+p)^{2}}} .
\end{aligned}
$$

The quantity under the radical has already been shown to be greater than unity; therefore,

$$
S_{a}>S_{b} \text { for } V \leqq W_{a} .
$$


For $V=\sqrt{p n} R, V / W_{a}$ is greater than unity, but $V / W_{b}$ is less than unity, and the sensitivities are, from eq 10 and 11:

$$
\begin{aligned}
& S_{a}=K \frac{\sqrt{n R}}{1+n} \times 1, \\
& S_{b}=K \frac{\sqrt{p R}}{1+p} \times \frac{\sqrt{p n} R}{p R(1+n)}(1+p)=K \frac{\sqrt{n R}}{1+n} .
\end{aligned}
$$

That is,

$$
S_{a}=S_{b} \text { for } V==\sqrt{p n} R .
$$

Now it is evident that for any value of $V$ less than $\sqrt{p n} R, S_{b}$ will be less than $K \sqrt{n R} / 1+n$, while for any value of $V$ between $W_{a}$ and $\sqrt{p n} R, S_{a}$ is constant and equal to $K \sqrt{n R} / 1+n$. Therefore,

$$
S_{a}>S_{b} \text { for } W_{a}<V<\sqrt{p n} R .
$$

For values of $V$ greater than $\sqrt{p n} R, S_{b}$ will be greater than $K \sqrt{n R} / 1+n$, while $S_{a}$ remains constant at $K \sqrt{n R} / 1+n$. Therefore,

$$
S_{b}>S_{a} \text { for } V>\sqrt{p n} R,
$$

completing the proof.

Washington, January 2, 1948 